\title{
Laboratory Monitoring in Emicizumab-Treated Persons with Hemophilia A
}

\author{
Jens Müller ${ }^{1,2}$ Isabell Pekrul ${ }^{3,4}$ Bernd Pötzsch ${ }^{1,2}$ Beate Berning ${ }^{5} \quad$ Johannes Oldenburg ${ }^{1,2}$ \\ Michael Spannagl ${ }^{4}$
}

1 Institute of Experimental Hematology and Transfusion Medicine, University of Bonn, Bonn, Germany

${ }^{2}$ Center for Rare Diseases Bonn (ZSEB), University Clinic Bonn, Bonn, Germany

${ }^{3}$ Department of Anaesthesiology, Ludwig Maximilian University (LMU) of Munich, Munich, Germany

${ }^{4}$ Department of Transfusion Medicine, Cell Therapeutics and Hemostaseology, Ludwig Maximilian University (LMU) of Munich, Munich, Germany

5 Roche Pharma AG, Grenzach-Wyhlen, Germany

Thromb Haemost 2019;119:1384-1393.

\author{
Address for correspondence Michael Spannagl, MD, PhD, \\ Department of Transfusion Medicine, Cell Therapeutics and \\ Hemostaseology, Ludwig Maximilian University of Munich, Munich, \\ Germany (e-mail: michael.spannagl@med.uni-muenchen.de).
}

\begin{abstract}
Keywords

- hemophilia A

- laboratory monitoring

- FVIII activity

- coagulation

Hemophilia A (HA) is an X-linked hereditary bleeding disorder caused by deficiency of coagulation factor $(F)$ VIII activity. One of the greatest complications in the treatment of HA is the development of neutralizing alloantibodies, known as FVIII inhibitors. HA patients who develop FVIII inhibitors have limited treatment options available to them and experience greater disease- and treatment-related burdens than HA patients without FVIII inhibitors. Emicizumab, a recently approved bispecific monoclonal antibody, mimics the function of FVIIla by bridging FIXa and FX to restore effective hemostasis. Although emicizumab and FVIII show some functional similarities, several key differences influence the results of standard laboratory assays when conducted in the presence of emicizumab, and can result in a misleading interpretation of coagulation assays in emicizumab-treated patients. Here, we discuss current laboratory monitoring methods, including activated partial thromboplastin time, FVIII one-stage clotting assays, FVIII chromogenic assays, and global coagulations assays; address why these conventional methods may be inappropriate for monitoring of HA patients receiving emicizumab; and suggest alternative methods applicable to monitoring $\mathrm{HA}$ treatment in an evolving treatment landscape.
\end{abstract}

\section{Introduction}

Hemophilia A (HA) is an X-linked hereditary bleeding disorder caused by deficiency of coagulation factor (F) VIII activity. Under physiological conditions, FVIII is activated by thrombin and subsequently acts as a cofactor for activated FIX (FIXa) to facilitate the activation of FX. Consequently, reduced FVIII activity leads to diminished generation of FXa, resulting in insufficient coagulation potential and bleeding complications. HA has three classifications determined by FVIII activity levels: mild HA (5 to $<40 \%$ FVIII activity); moderate HA $(1$ to $<5 \%)$; and severe $\mathrm{HA}(<1 \%) .{ }^{1}$ Usually, $\mathrm{HA}$ is treated via the intravenous administration of recombinant or plasma-derived FVIII concentrates to restore hemostasis. Recombinant factor administration is required every 2 to 3 days due to the approximate 12-hour half-life of FVIII. Recent advances in technology have led to the development of extended half-life (EHL) factor products, resulting received

February 8, 2019

accepted after revision

April 28, 2019
DOI https://doi.org/

10.1055/s-0039-1692427.

ISSN 0340-6245.
@ 2019 Georg Thieme Verlag KG
Stuttgart · New York

License terms

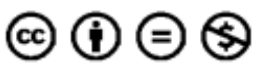


in reduced frequency of intravenous infusions and, consequently, reduced therapeutic burden. ${ }^{1}$

One of the greatest complications following FVIII replacement for the treatment of HA is the development of neutralizing alloantibodies against the replacement factor. These antibodies, also known as FVIII inhibitors, develop in 25 to $30 \%$ of persons with severe HA and render FVIII replacement therapies ineffectual, thus exposing HA patients to a greater risk of spontaneous and traumatic bleeding episodes. ${ }^{2} \mathrm{HA}$ patients who develop FVIII inhibitors have limited treatment options available to them; bypassing agents (BPAs), including activated prothrombin complex concentrate and recombinant activated FVII, offer alternative treatment options but are associated with a greater burden of treatment and inconsistent bleeding control. ${ }^{3}$ Compared with those without FVIII inhibitors, HA patients with FVIII inhibitors are at greater risk of disease-related morbidity and mortality. ${ }^{4}$

A new treatment option for HA patients with FVIII inhibitors is subcutaneous $1.5 \mathrm{mg} / \mathrm{kg}$ once-weekly administration of emicizumab (HEMLIBRA; F. Hoffmann-La Roche, Basel, Switzerland), a novel, bispecific, humanized, monoclonal antibody, which has been approved in several countries ${ }^{5,6}$ for routine prophylaxis for HA patients with FVIII inhibitors. This initial approval has now been expanded to include prophylaxis of 1.5 $\mathrm{mg} / \mathrm{kg}$ emicizumab once-weekly, $3.0 \mathrm{mg} / \mathrm{kg}$ once every two weeks, or $6.0 \mathrm{mg} / \mathrm{kg}$ every four weeks, irrespective of inhibitor status (although the European Medicines Agency has approved emicizumab for use in patients without inhibitors only for those with severe $\left[<1 \%\right.$ FVIII activity] HA). ${ }^{5,6}$ Emicizumab mimics the function of FVIIIa by bridging FIXa and FX to restore effective hemostasis. ${ }^{7}$ There is no evidence to suggest that emicizumab induces the development of FVIII inhibitors, as expected given the lack of structural homology between FVIII and emicizumab. ${ }^{8}$ Risk of FVIII inhibitor development in patients treated with emicizumab who receive infrequent doses of FVIII for breakthrough bleeds or surgery continues to be monitored in long-term follow-up. Results from the phase III clinical trials in HA patients with FVIII inhibitors and HA patients without FVIII inhibitors have previously been published. ${ }^{9-12}$

Here, we discuss current laboratory monitoring methods used during the management of HA, why these conventional methods may be inappropriate for monitoring of HA patients receiving emicizumab, and alternative methods for monitoring HA treatment in an evolving treatment landscape.

\section{Current Methods in the Coagulation Laboratory}

Current laboratory methods are comprised of coagulation tests and chromogenic substrate assays (CSAs), which ultimately aim to assess the coagulation potential of the patient.

Coagulation assays used for FVIII testing are based on the format of activated partial thromboplastin time (aPTT). The aPTT measures the time from standardized activation of the intrinsic coagulation pathway by a contact-phase activator (ellagic acid, silica, or kaolin) to fibrin clot formation after the final addition of a calcium chloride solution as the starting reagent.

One common laboratory test based on the aPTT is the one-stage FVIII clotting assay (OSA), which measures the ability of sample patient plasma to shorten the aPTT after mixing it with FVIII-deficient plasma. ${ }^{13,14}$ The mixture of FVIII-deficient plasma and the patient sample are incubated with the aPTT reagent containing a contact-phase activator and phospholipids, before calcium chloride is added to initiate clot formation. In this assay, FVIII concentration is considered to be the rate-determining step of measured clotting time (CT). ${ }^{14}$ The FVIII OSA is the most widely used coagulation assay, and can be used to measure even very low levels of plasma FVIII activity. ${ }^{15}$ However, OSA results are subject to interlaboratory variation, due in part to the wide range of aPTT reagents available and clot detection techniques used. ${ }^{14}$

An alternative to the FVIII OSA is the two-stage FVIII clotting assay, which was first developed in 1955 as a modified thromboplastin dilution test. ${ }^{13,16}$ As with the FVIII OSA, FVIII concentration is the rate-determining step in the two-stage assay, but despite being less susceptible to reagent variation, this assay is not commonly used in the clinical setting due to its complexity.

Based on a similar principle to the two-stage clotting assay, the FVIII CSA measures the FVIII-dependent activation of FX using purified human or bovine coagulation factors. $^{13,17}$ In the first stage of this assay, patient plasma is added to a reaction mixture that usually contains FIXa, FX, calcium ions, phospholipids, and trace amounts of thrombin. Thrombin triggers the activation of FVIII and the subsequent FIXa-mediated activation of FX. In contrast to the FVIII OSA, the addition of thrombin in the first step means that activation of FVIII to FVIIIa is not a major influencing determinant of the assay; FXa production is presumed to be proportional to the FVIII concentration in the plasma sample. The step that distinguishes the FVIII CSA from the original two-stage assay is the addition of a FXa-selective chromogenic peptide substrate in the second phase of the assay to measure the amount of FXa formed during the first phase of the assay. Increased absorbance due to cleavage of the substrate is usually measured at a wavelength of $405 \mathrm{~nm} .^{13,17}$

In comparison to the FVIII OSA, the FVIII CSA procedure is more standardized and, as such, offers higher interlaboratory precision in the measurement of plasma FVIII activity. Despite the benefits of the CSA, it is considered to be a comparatively complex and expensive assay to perform in the clinical setting. ${ }^{18}$ However, the costs of the CSA can be reduced through batch testing and by aliquoting and freezing of reconstituted reagents. ${ }^{18}$

FVIII CSAs and OSAs often provide discordant measures of FVIII activity; during diagnosis, discrepancies between these two assays can lead to up to $10 \%$ of patients with mild HA being misdiagnosed. ${ }^{16,19-21}$ With respect to the monitoring of FVIII replacement therapy, the FVIII OSA tends to accurately measure plasma-derived and full-length recombinant FVIII concentrates. However, it has been known that, depending on the aPTT reagent used, the true activity 
of B-domain deleted and/or EHL products may be underestimated by up to $50 \%$ when compared with the FVIII CSA that, in accordance with the European Pharmacopoeia, is used for the potency labeling of FVIII concentrates approved under the aegis of the European Medicines Agency. ${ }^{22}$ If the FVIII CSA is not available, the FVIII OSA may be used for the accurate measurement of these factors through the application of product-specific standards (as for ReFacto) or established conversion factors (as proposed for Afstyla). ${ }^{23,24}$

In addition to aPTT, other global coagulation assays include rotational thromboelastometry (TEM) (ROTEM; Pentapharm GmbH, Munich, Germany) and the thrombin generation assay (TGA), which can provide a more extensive analysis of hemostatic activity.

ROTEM is a well-established and increasingly prominent set of tests characterizing viscoelastic parameters of clot formation in whole blood. ${ }^{25,26}$ There are several different assays used with a ROTEM instrument, classed as activated TEM (including intrinsic pathway [IN]TEM and extrinsic pathway [EX]TEM) or nonactivated TEM (native coagulation [NA]TEM). ${ }^{27}$ Data suggest that the NATEM method is more sensitive to changes in coagulation than the activated counterparts. $^{28}$ The parameters of clot formation defined by ROTEM are CT, clot formation time (CFT), maximum clot firmness (MCF), and the clot lysis index. ${ }^{26}$

The TGA is usually triggered by tissue factor (TF) (extrinsic pathway) or FXIa (intrinsic pathway), leading to thrombin generation, which is monitored over time. The derived curves are comprised of five parameters: lag time, endogenous thrombin potential, thrombin peak, time to peak, and velocity index. ${ }^{29}$ While the TGA is increasingly used as a measure of global coagulation in patients with bleeding disorders, this method is yet to be standardized and there is great interlaboratory variation. ${ }^{29,30}$

Monitoring of hemostasis also includes the determination of the presence of FVIII inhibitors. In general, inhibitor assays compare the residual FVIII activity in a mixture containing a patient plasma sample and a source of FVIII (e.g., normal pooled plasma [NPP]) with the residual FVIII activity in a mixture of diluent and NPP, as measured by the FVIII OSA; this comparison permits the quantification of FVIII activity reduction by FVIII inhibitors and allows the analysis of FVIII inhibitor titers. ${ }^{31}$ In 1975, the inhibitor assay was standardized. Known as the Bethesda assay, this standardized method defines one Bethesda unit as the amount of inhibitor required in $1 \mathrm{~mL}$ of sample plasma to reduce the FVIII activity in $1 \mathrm{~mL}$ of NPP by $50 \%$ in 2 hours at $37^{\circ} \mathrm{C} .{ }^{31}$ Since then, the Bethesda assay has evolved to include imidazole buffering of the NPP, and the use of FVIII-deficient plasma rather than buffer in the control mixture and for dilution of high-titer inhibitor plasma (Nijmegen-Bethesda Assay [NBA]). ${ }^{31}$ Heating of patient plasmas to $56^{\circ} \mathrm{C}$ for 30 minutes has been shown to separate antigen-antibody complexes and denature FVIII, so allowing for the accurate assessment of FVIII inhibitor status in HA patients receiving FVIII products. ${ }^{32}$

Clot-based Bethesda assays are responsive to unspecific inhibitors of coagulation such as antiphospholipid antibo- dies showing a lupus anticoagulant phenotype and anticoagulants such as heparin, in addition to specific FVIII inhibitors. This nonspecific sensitivity makes it difficult to distinguish between general inhibition of CT and FVIIIspecific inhibition. ${ }^{31}$ As the FVIII CSA has a more specific endpoint (FXa cleavage), it is not responsive to general coagulation inhibitors and, thus, allows for more specific measurement of FVIII activity. Accordingly, a chromogenic Bethesda Assay (CBA) has been described. This assay is identical to the conventional NBA except for the incorporation of a FVIII CSA to determine residual FVIII activity. ${ }^{31}$ The CBA is therefore more specific to FVIII inhibitors than the original NBA. ${ }^{31}$

An enzyme-linked immunosorbent assay (ELISA) can also be used to detect antibodies to FVIII. ${ }^{33,34}$ The ELISA may be more sensitive to the presence of FVIII alloantibodies than the NBA, but it cannot distinguish between clinically relevant inhibitory antibodies and noninhibitory antibodies. As such, ELISA and the NBA may be applied in combination; the ELISA is used for the initial detection of antibodies of any form, and the Bethesda assay is then used to confirm the antibody as inhibitory. ${ }^{31}$

\section{FVIII and Emicizumab: Similarities and Differences}

While emicizumab mimics the activity of FVIIIa, the two molecules have no structural homology and have several biochemical and functional differences ( - Table 1 ).

The emicizumab clinical trials have highlighted further differences between emicizumab and FVIII. Emicizumab has a half-life of approximately 30 days ${ }^{5,6,35,36}$ and has been shown to have achieved stable mean plasma concentrations by treatment week 4 in the clinical studies. ${ }^{9-12}$ Conversely, FVIII has a half-life of approximately 12 hours, ${ }^{37}$ dependent on several inter- and intraindividual factors. Due to the prolonged half-life of emicizumab, stable mean plasma concentrations of approximately $40 \mu \mathrm{g} / \mathrm{mL}$ are achieved even with administration of $6 \mathrm{mg} / \mathrm{kg}$ emicizumab once every four weeks, providing HA patients with prolonged protection from traumatic and spontaneous bleeding events. ${ }^{11}$ Furthermore, no association between emicizumab and the development of de novo FVIII inhibitors has been found in any of the pivotal phase III clinical trials, nor is emicizumab activity affected by the presence of FVIII inhibitors. ${ }^{9-12}$

All of the above differences between FVIII and emicizumab influence the results of several standard laboratory assays when conducted in the presence of emicizumab, and can result in a misleading interpretation of coagulation assays in emicizumab-treated patients. Moreover, the bleeding rate during emicizumab prophylaxis observed in the clinical studies could suggest reversion to a mild hemophilia phenotype. Yet, we do not know whether this correlation holds true in all situations (during surgery, after trauma); the mechanism of action of emicizumab is distinct from FVIII, therefore, direct correlation to FVIII activity is not conclusive and may lead to misinterpretation. ${ }^{38}$ 
Table 1 A comparison of the properties of FVIII and emicizumab

\begin{tabular}{|c|c|}
\hline FVIII & Emicizumab \\
\hline $\begin{array}{l}\text { FVIII is a heterodimeric protein with a molecular weight of } \\
\sim 280 \text { kDa and a normal plasma concentration of } \sim 0.4 \mathrm{nM} .{ }^{44} \\
\text { The half-life of FVIII is } \sim 12 \text { hours }\end{array}$ & $\begin{array}{l}\text { Emicizumab is a humanized bispecific monoclonal antibody } \\
\text { with a molecular weight of } \sim 150 \mathrm{kDa} \text {. The target plasma } \\
\text { concentration for prophylactic treatment of HA patients is } \\
\sim 0.4 \mu \mathrm{M} \text {. The half-life of emicizumab is } \sim 30 \text { days } 35,36\end{array}$ \\
\hline $\begin{array}{l}\text { FVIII must be activated by thrombin-mediated proteolysis to } \\
\text { obtain its cofactor activity. The activity of FVIIla is regulated by } \\
\text { spontaneous dissociation of the A2 domain as well as inacti- } \\
\text { vation by activated protein C. Thus, FVIII exhibits on/off } \\
\text { mechanisms } 44\end{array}$ & $\begin{array}{l}\text { Emicizumab does not require an activation step to facilitate its } \\
\text { activity and there is no inactivation of the molecule. Thus, } \\
\text { there are no on/off mechanisms for emicizumab }{ }^{36,44}\end{array}$ \\
\hline $\begin{array}{l}\text { FVIIla has high affinity for its target factors. In the absence of a } \\
\text { phospholipid bilayer, FVIIla binds to FIXa with an affinity of } \\
\sim 15 \mathrm{nM} \text {, and to FX with an affinity of } \sim 0.3 \mu \mathrm{M} \text {. In contrast, } \\
\text { FVIIla shows only weak-to-no binding to FIX }{ }^{64} \text { and FXa. }{ }^{65,66} \\
\text { FVIIla binds as well to human as to bovine FIXa and FX }\end{array}$ & $\begin{array}{l}\text { Emicizumab does not distinguish between the precursor and } \\
\text { activated forms of its target factors. This is to say that } \\
\text { emicizumab has a similar affinity for the precursors FIX and FX } \\
\text { and for the activated factors FIXa and FXa ( } 1.6 \text { and } 1.9 \mu \mathrm{M} \text { vs. } \\
1.5 \text { and } 1.0 \mu \mathrm{M} \text {, respectively). }{ }^{35} \text { Emicizumab binds to human } \\
\text { but not to bovine FIX(a) and FX(a) }\end{array}$ \\
\hline $\begin{array}{l}\text { FVIIla shows full cofactor activity; it promotes binding of FIXa } \\
\text { to phospholipids, stabilizes the active site of FIXa, and finally } \\
\text { bridges FIXa to FX }{ }^{44}\end{array}$ & $\begin{array}{l}\text { Emicizumab shows partial cofactor activity by bridging FIXa to } \\
\text { FX. The process also depends on properly aligned target } \\
\text { proteins on the phospholipid surface } 44\end{array}$ \\
\hline $\begin{array}{l}\text { The plasma concentration of FVIII }(\sim 0.4 \mathrm{nM}) \text { is significantly } \\
\text { lower than the concentrations of FIX and FX }(\sim 90 \text { and } 135 \mathrm{nM} \text {, } \\
\text { respectively). This implies that tenase activity is limited by the } \\
\text { amount of FVIIIla generated }{ }^{44}\end{array}$ & $\begin{array}{l}\text { Prophylactic treatment with emicizumab aims for a target } \\
\text { concentration of } \sim 0.4 \mu \mathrm{M} \text {, much greater than the plasma } \\
\text { concentrations of endogenous FX and FIXa. With emicizumab } \\
\text { in excess of its target proteins, the rate-limiting step for } \\
\text { activation of FX is the amount of FIXa generated }\end{array}$ \\
\hline $\begin{array}{l}\text { As a cofactor, FVIIla facilitates the activation of FX by FIXa by a } \\
\text { factor of } \sim 10^{6} .44 \text { Thus, the catalytic efficiency of FIXa in the } \\
\text { presence of FVIIIla is } \sim 11 \text {-fold higher than that of FIXa in the } \\
\text { presence of emicizumab }\end{array}$ & $\begin{array}{l}\text { As a cofactor, emicizumab facilitates the activation of FX by } \\
\text { FIXa by a factor of } \sim 9 \times 10^{4} \text {. Thus, the catalytic efficiency of } \\
\text { FIXa in the presence of emicizumab is } \sim 11 \text {-fold lower than } \\
\text { that of FIXa in the presence of FVIIIa } 35\end{array}$ \\
\hline
\end{tabular}

Abbreviations: F, coagulation factor; HA, hemophilia A.

\section{Impact and Interference of Emicizumab on Current Laboratory Assays}

The established structural and functional dissimilarities between FVIII and emicizumab cause several interactions and interferences with the monitoring assays outlined in - Table 2.

As discussed previously, aPTT measures the time from activation of the intrinsic coagulation pathway to fibrin clot formation. As emicizumab does not require activation by thrombin to facilitate the propagation of the clotting cascade, a major rate-limiting step is eliminated and aPTT appears to normalize at subtherapeutic emicizumab concentrations. ${ }^{5,6,39}$

Given the long half-life of emicizumab, residual interference on aPTT can continue for up to 6 months after discontinuation of treatment. ${ }^{5,6}$ In general, those performing aPTT-based assays must have knowledge of the fact that the patient's sample contains emicizumab to allow accurate interpretation of the assay results. ${ }^{40}$ While this is likely to be the case in hemophilia treatment centers where routine monitoring of hemophilia takes place, it is recommended that HA patients treated with emicizumab carry an emergency alert card. Emergency caregivers (e.g., hospital staff) should be aware of the influence of emicizumab on aPTT (i.e., giving the appearance of full coagulation restoration even at low levels), and the aPTT-based OSA. Misinterpretation of assay results would raise safety risks such as carrying out surgical procedures when it is not safe to do so, and insufficient treatment of serious bleeding events.

As a result of the interference of emicizumab with aPTT, FVIII OSAs report FVIII activities exceeding $150 \%$ in the presence of emicizumab. ${ }^{5,6,16,36}$ Furthermore, the interference of emicizumab on aPTT renders all aPTT-based assays inaccurate, regardless of the reagents used. ${ }^{41,42}$ Consequently, all variations of OSAs and Bethesda assays using OSA to interpret results should be avoided in the management of patients receiving emicizumab.

Interference analyses have shown that emicizumab does not exert a similar effect on non-aPTT-based assays, such as thrombin time and fibrinogen assays, and does not influence the extrinsic coagulation pathway and the regulation of hemostatic factors therein. ${ }^{40}$ As such, the global coagulation assays ROTEM and the TGA are thought to accurately measure coagulation in the presence of emicizumab. ${ }^{25,42}$

Chromogenic assays, including antithrombin activity, protein $C$ activity, and plasminogen activity, are not influenced by the presence of emicizumab and continue to give accurate results, with the exception of the FVIII CSA using human-derived components, in which emicizumab affects the reported FVIII activity. ${ }^{40}$

Emicizumab has varied effects on FVIII inhibitor detection assays. As emicizumab is unaffected by the presence of FVIII 
Table 2 A summary of the interactions and interferences of emicizumab with commonly used monitoring assays

\begin{tabular}{|c|c|c|c|}
\hline & Standard assay & Sensitivity to FVIII/emicizumab & $\begin{array}{l}\text { Recommendations for use in } \\
\text { the presence of emicizumab }\end{array}$ \\
\hline \multirow[t]{3}{*}{$\begin{array}{l}\text { Global coagulation } \\
\text { assays }\end{array}$} & aPTT & $\begin{array}{l}\text { FVIII: sensitive } \\
\text { Emicizumab: oversensitive; nor- } \\
\text { malizes at very low concentrations } \\
\text { of emicizumab } 39\end{array}$ & $\begin{array}{l}\text { Cannot be used to measure the } \\
\text { hemostatic potential of a } \\
\text { patient }\end{array}$ \\
\hline & ROTEM & $\begin{array}{l}\text { FVIII: sensitive } \\
\text { Emicizumab: sensitive; EXTEM has } \\
\text { little-to-no response in the presence } \\
\text { of emicizumab and INTEM shows } \\
\text { marked response in the presence of } \\
\text { emicizumab, but no dose-depen- } \\
\text { dent effect, while NATEM shows a } \\
\text { significant dose-dependent } \\
\text { response to emicizumab } 52\end{array}$ & $\begin{array}{l}\text { May be used to measure global } \\
\text { coagulation in the presence of } \\
\text { emicizumab }\end{array}$ \\
\hline & TGA & $\begin{array}{l}\text { FVIII: sensitive } \\
\text { Emicizumab: sensitive; shows a lin- } \\
\text { ear, dose-dependent increase in } \\
\text { thrombin generation when trig- } \\
\text { gered with FXIa }{ }^{48}\end{array}$ & $\begin{array}{l}\text { May be used to measure global } \\
\text { coagulation in the presence of } \\
\text { emicizumab }\end{array}$ \\
\hline \multirow[t]{4}{*}{ FVIII activity assays } & FVIII OSA (aPTT-based) & $\begin{array}{l}\text { FVIII: sensitive } \\
\text { Emicizumab: oversensitive; nor- } \\
\text { malizes at very low concentrations } \\
\text { of emicizumab } 16,36\end{array}$ & $\begin{array}{l}\text { Cannot be used to measure } \\
\text { emicizumab or FVIII activity }\end{array}$ \\
\hline & $\begin{array}{l}\text { Modified FVIII OSA calibrated } \\
\text { against emicizumab }\end{array}$ & $\begin{array}{l}\text { FVIII: slightly sensitive } \\
\text { Emicizumab: sensitive }\end{array}$ & $\begin{array}{l}\text { May be used to measure emici- } \\
\text { zumab plasma levels }\end{array}$ \\
\hline & $\begin{array}{l}\text { FVIII CSA (bovine compo- } \\
\text { nents: Siemens, etc.) }\end{array}$ & $\begin{array}{l}\text { FVIII: sensitive } \\
\text { Emicizumab: insensitive; emicizu- } \\
\text { mab does not accelerate FXa for- } \\
\text { mation by bovine FIXa } 57\end{array}$ & $\begin{array}{l}\text { Cannot be used to measure } \\
\text { emicizumab activity. May be } \\
\text { used to measure FVIII activity } \\
\text { without interference from emi- } \\
\text { cizumab in the sample }\end{array}$ \\
\hline & $\begin{array}{l}\text { FVIII CSA (human compo- } \\
\text { nents: Hyphen BIOPHEN) }\end{array}$ & $\begin{array}{l}\text { FVIII: sensitive } \\
\text { Emicizumab: sensitive (within the } \\
\text { dynamic range of the assay) }{ }^{41}\end{array}$ & $\begin{array}{l}\text { Reported FVIII cannot be viewed } \\
\text { as equivalent to FVIII activity } \\
\text { measured in patients treated } \\
\text { with FVIII. May only provide a } \\
\text { relative indication of the pro- } \\
\text { coagulant activity of } \\
\text { emicizumab }\end{array}$ \\
\hline \multirow[t]{2}{*}{ Inhibitor assays } & $\begin{array}{l}\text { Bethesda assays using FVIII } \\
\text { OSA }\end{array}$ & $\begin{array}{l}\text { FVIII: sensitive } \\
\text { Emicizumab: false negative; as } \\
\text { emicizumab is not deactivated by } \\
\text { heat, it drives coagulation via } \\
\text { human FIX and FX in human plasma, } \\
\text { regardless of presence of inhibitors } \\
\text { to FVIII }\end{array}$ & $\begin{array}{l}\text { Cannot be used to measure FVIII } \\
\text { inhibitor titers in the presence of } \\
\text { emicizumab }\end{array}$ \\
\hline & CBA & $\begin{array}{l}\text { FVIII: sensitive } \\
\text { Emicizumab: insensitive; this assay } \\
\text { uses a bovine protein-based chro- } \\
\text { mogenic FVIII assay, which is not } \\
\text { affected by emicizumab }{ }^{42}\end{array}$ & $\begin{array}{l}\text { Can be used to measure FVIII } \\
\text { inhibitor titers in the presence of } \\
\text { emicizumab }\end{array}$ \\
\hline
\end{tabular}

Abbreviations: aPTT, activated partial thromboplastin time; CBA, chromogenic Bethesda Assay; CSA, chromogenic assay; F, coagulation factor; OSA, one-stage clotting assay.

inhibitors and is insensitive to heat treatment (i.e., it retains its full activity), the FVIII OSA-based NBA will return artificial (false-negative) results when emicizumab is present. ${ }^{31}$ However, immunoassays, including ELISA, are not affected by the presence of emicizumab in the test sample and can be used to monitor FVIII alloantibodies in the presence of emicizumab.

\section{Assay Alternatives}

Due to the well-documented interference of emicizumab on many of the commonly used hemophilia monitoring assays, alternative methods are required (and available) for the measurement of emicizumab plasma concentrations and 
FVIII activities, including the determination of FVIII inhibitor titers in emicizumab-treated patients.

\section{Measurement of Emicizumab}

In a preclinical study of BS23, the precursor for emicizumab, FVIII-like activity was extrapolated from emicizumab concentrations in an attempt to identify potential efficacious dosing regimens for use in the later clinical trials. ${ }^{7}$ With improved understanding of the characteristics of emicizumab, and as the differences between emicizumab and FVIII became more apparent, it was accepted that FVIII-like activity was not appropriate for this task and efficacious emicizumab concentrations, as used in the HAVEN clinical studies, were instead estimated from the first in vivo studies of emicizumab. ${ }^{39,43}$ Due to the mechanistic differences between emicizumab and FVIII, the aim of hemostatic monitoring in the presence of emicizumab should be to determine emicizumab plasma concentrations rather than "equivalent" FVIII activity. Moreover, FVIII-like activities that correspond to the therapeutic dose of emicizumab will vary across coagulation assays. For example, in the aPTT-based FVIII assay, $0.1 \mathrm{U} / \mathrm{mL}$ FVIII activity corresponds to $4.0 \mathrm{nM}$ emicizumab, but matches $250 \mathrm{nM}$ emicizumab in TF-based TGAs and $500 \mathrm{nM}$ emicizumab in FXIa-induced TGAs. ${ }^{44}$ The assays were developed with FVIII as the limiting factor, which is not the case for emicizumab as it is present in excess to the relevant components. Rather, the FIXa levels generated in these assay systems determine the apparent activity of emicizumab, leading to apparent disparities between assays. ${ }^{45}$

A modified version of the traditional aPTT-based FVIII OSA ( $\mathrm{r}^{2}$ Diagnostics, South Bend, Indiana, United States), which has been calibrated against emicizumab and includes a dedicated plasma emicizumab calibrator as well as plasma-based controls, has recently been developed and commercialized in many countries including Germany, Switzerland, and Austria (distributed by Haemochrom Diagnostica GmbH, Essen, Germany) to enable the accurate measurement of plasma emicizumab concentration. ${ }^{46}$ This assay follows the same method as the traditional FVIII OSA, but is instead calibrated against emicizumab and deploys a higher predilution of the patient sample (1:8) to enable a dynamic range between 10 and 100 $\mu \mathrm{g} / \mathrm{mL}$ of emicizumab in the original plasma sample. In a recent analysis, this novel method was shown to have excellent precision and reproducibility. ${ }^{46}$ In a second analysis, samples containing emicizumab analyzed with the modified FVIII OSA showed emicizumab levels that significantly correlated with the noncommercial ELISA method previously used in the HAVEN trials. ${ }^{41}$ Results to date indicate that the modified FVIII OSA may be used to establish whether or not emicizumab plasma concentrations are within the previously determined efficacious range (30-70 $\mu \mathrm{g} / \mathrm{mL}$; efficacy does not increase further above mean plasma concentrations of $\geq 25-30 \mu \mathrm{g} /$ $\mathrm{mL}){ }^{47}$ Currently, this assay is recognized as the easiest and most accurate method by which to monitor emicizumab concentration in plasma.

While a FVIII CSA utilizing human-derived FIXa and FX is responsive to the presence of emicizumab, ${ }^{41}$ this assay is not recommended for use as a measure of emicizumab activity.
Despite the observed linear correlation with emicizumab plasma levels, ${ }^{48}$ CSA-reported FVIII activity is not directly equivalent to emicizumab levels, and comparison of these activities should be performed for qualitative purposes only and should be conducted with caution. Furthermore, reported FVIII-like activity is also influenced by any FVIII in the sample and may not be specifically attributed to either FVIII or emicizumab. However, the influence of FVIII may be eliminated by heat treatment of the plasma sample to improve the sensitivity of the assay for emicizumab. Furthermore, the available emicizumab plasma calibrator and controls applied in the modified FVIII OSA for measurement of plasma emicizumab levels may, in theory, also be used in conjunction with the FVIII CSA based on human factors. To date, however, no data on the performance of such an assay combination are available.

In the clinical setting, the modified FVIII OSA calibrated against emicizumab may be used to monitor emicizumab plasma concentrations in HA patients. Plasma concentrations can be matched to the effective range of emicizumab, which showed a sufficient hemostatic potential in clinical trials. ${ }^{9-12,49}$ However, the reported efficacy of emicizumab in the recent HAVEN clinical trials ${ }^{9-12}$ suggests that such treatment does not need to be monitored routinely in the clinical setting, except for in the case of breakthrough bleeding and subsequent treatment with on-demand coagulation factors, surgical procedures, or inhibitor monitoring. ${ }^{50}$

With regard to global coagulation assays, both ROTEM and TGA can be used to monitor hemostasis in emicizumabtreated patients, although these assays are not used widespread in the clinical setting. . $^{2,42}$

ROTEM (NATEM) was used to monitor the $\mathrm{Ca}^{2+}$-triggered hemostatic functions of patients treated with emicizumab initially in a phase I trial of Japanese patients. ${ }^{51}$ Here, samples spiked with emicizumab ex vivo were shown to have improved hemostatic function in an emicizumab dosedependent manner, irrespective of the presence of FVIII inhibitors. ${ }^{51}$ More recently, nonactivated ROTEM methods (NATEM) have been generally shown to be more informative in terms of the global coagulation response to emicizumab than the activated EXTEM and INTEM methods. ${ }^{52}$ In this analysis, EXTEM was shown to have little-to-no response in the presence of emicizumab, and while INTEM demonstrated a marked response to the presence of emicizumab, there was no significant difference between results at different concentrations of emicizumab. NATEM, on the other hand, showed a significant dose-dependent response to emicizumab. This effect was most noticeable in terms of CT and CFT, while $\alpha$-angle and MCF were less indicative of hemostatic activity in this study. ${ }^{52}$ While the sensitivity of ROTEM to emicizumab has been demonstrated through the use of both ex vivo spiking studies and the analysis of postinfusion samples, there is some evidence to suggest that analyzing samples spiked with emicizumab ex vivo overestimates hemostatic function, ${ }^{53}$ and further research into the accuracy of ROTEM in emicizumab-treated patients is required.

The TGA shows a linear, dose-dependent increase in thrombin generation in the presence of emicizumab, ${ }^{48}$ 
and, as such, can be used to monitor the global hemostatic response in patients treated with emicizumab. TGA has been demonstrated to be more responsive to emicizumab when FXIa is used as the trigger rather than $\mathrm{TF}^{54}$ Moreover, emicizumab has been shown to have similar activity to FVIII in TGA. ${ }^{55}$ Additionally, the TGA can be used to monitor the cumulative effect of emicizumab and BPAs in patients with FVIII inhibitors, and can therefore be used as a tool to individually tailor BPA therapy. ${ }^{56}$ This is of particular interest as patients treated with emicizumab are expected to continue to use BPAs for the treatment of breakthrough bleeding events, as in the HAVEN clinical trials. ${ }^{9-12}$

\section{Measurement of FVIII Activity in the Presence of Emicizumab}

As discussed above, emicizumab selectively binds to human FIXa and FX. Thus, a FVIII CSA based on bovine factors is unaffected by the presence of emicizumab, as FIXa and FX in the reaction mixture will only bind to endogenous or infused FVIII in the sample. This is to say that FVIII activity, as measured by the bovine FVIII CSA, reflects the FVIII activity in the sample and does not account for the coagulation potential of emicizumab. Therefore, the bovine assay continues to provide an accurate measurement of endogenous or infused FVIII activity independent of emicizumab. ${ }^{57}$ This assay may be particularly useful in cases where emicizumab and FVIII are used in parallel, such as following trauma, during surgery, or during immune tolerance induction.

Where a FVIII CSA based on bovine factors is not available, there is no easy way to monitor FVIII independently of emicizumab. In Japan, anti-idiotype emicizumab monoclonal antibodies (mAbs) have been suggested as an alternative route for the measurement of FVIII activity in patients receiving emicizumab by rendering the antibody inactive and eliminating its capacity to interfere with traditional aPTT-based clotting assays. ${ }^{58}$ Two anti-idiotype antibodies were developed (rcAQ8, a mAb binding to the anti-FIXa arm of emicizumab, and rcAJ540, an anti-FX arm $\mathrm{mAb}$ ) and assessed using aPTT and FVIII OSA; the authors concluded that the addition of both rcAQ8 and rcAJ540 to samples containing emicizumab almost completely eliminated its binding potential for human FIXa and FX, meaning the assays used measured FVIII activity as they would in the absence of emicizumab. ${ }^{58}$ However, it is currently unknown whether these assays will be commercialized outside of Japan, Korea, and Taiwan.

\section{Measurement of FVIII Inhibitors in the Presence of Emicizumab}

In the absence of emicizumab, the NBA based on a FVIII OSA is most commonly used to determine the inhibitor status of HA patients receiving FVIII replacement; the CBA is rarely used in this setting due to higher costs and greater complexity. $5,6,42,59$ However, when based on a bovine FVIII CSA, the $\mathrm{CBA}$ is insensitive to emicizumab and accurately measures residual FVIII activity. ${ }^{42}$ This assay was used during the HAVEN study program to monitor FVIII inhibitor titers over time. $^{9-12,60}$
The previously discussed use of anti-idiotype antibodies to neutralize emicizumab can also be implemented in the FVIII OSA-based NBA, removing the influence of emicizumab and thus allowing the accurate measurement of FVIII inhibitor titers. ${ }^{58}$ Again, this method may not become widely available.

A solid-phase indirect anti-FVIII ELISA (e.g., LIFECODES FVIII antibody screen, Immucor GTI Diagnostics Inc., Waukesha, Wisconsin, United States; ZYMUTEST Anti-VIII IgG Mono-Strip, CoaChrom Diagnostica GmbH, Vienna), may be used to detect FVIII alloantibodies. ${ }^{61}$ The presence of emicizumab does not influence the results of this assay.

\section{Measurement of Anti-Emicizumab Antibodies}

During drug development, emicizumab was engineered to overcome identified product-related risk factors ${ }^{62}$ for antiemicizumab antibody (ADA) development via methods including humanization and bispecific immunoglobulin $G$ purification. ${ }^{8}$ While the immunogenicity of emicizumab is expected to be lower than that of FVIII, ${ }^{7}$ ADA development has been reported across the HAVEN clinical program. ${ }^{63}$ While ADAs are distinct from FVIII inhibitors, they also can be neutralizing or nonneutralizing, and have the potential to influence the efficacy, safety, and pharmacokinetics of emicizumab. Until now, no patient- or disease-related risk factors attributed to the increased likelihood of ADA development have been identified.

In the emicizumab clinical trials, ADAs in patient plasma were detected using an Food and Drug Administrationvalidated electrochemiluminescence immunoassay method (phase I only), or using ELISA (phase III). ${ }^{63}$ Using the ELISA method, 14 of 398 (3.5\%) participants tested positive for antiemicizumab ADAs; 7 of these cases were transient, while 3 were considered to have neutralizing potential. ${ }^{63}$

In the case that neutralizing ADAs are suspected, for example, in patients with increasing bleeding rates, noncompliance to the prescribed emicizumab regimen should first be ruled out as the cause. To do this, the next scheduled emicizumab dose could be administered at the patient's treatment center to allow for the monitoring of plasma emicizumab concentrations using the modified FVIII OSA calibrated against emicizumab following administration. If emicizumab plasma concentrations are not seen to increase following administration, the presence of neutralizing ADAs is inferred.

At present, no commercial assay for the direct identification of emicizumab ADAs is available. Instead, functional assays may be used to indirectly detect the possible presence of ADAs when suspected as the cause of reduced bleed control. For example, neutralizing ADAs have been shown to prolong the shortening effect of emicizumab on aPTT. ${ }^{36}$ When using aPTT-based clotting assays, such observations can imply the presence of inhibitory ADAs in the sample if the patient is adherent to treatment. Since aPTT normalizes at subtherapeutic emicizumab concentrations, aPTT will only be prolonged when more than $90 \%$ of emicizumab is neutralized by ADAs. Due to the higher predilution of the patient sample, a reduction in reported emicizumab plasma 
concentrations in the modified FVIII OSA calibrated against emicizumab might, therefore, indicate the presence of a neutralizing ADA earlier than standard aPTT tests.

However, as the incidence of ADAs with neutralizing potential is relatively low, ${ }^{63}$ routine monitoring of ADAs is not necessarily required. Even in circumstances when ADAs are identified, patients may remain on their prescribed emicizumab treatment and continue to achieve therapeutic bleed control. Across the HAVEN program, only one participant discontinued from emicizumab treatment due to loss of efficacy following development of ADAs with neutralizing potential. Of 398 participants, 13 others who tested positive for ADAs had antibodies classified as transient $(n=7)$, without neutralizing potential $(n=4)$, or antibodies with neutralizing potential that did not reduce the efficacy of emicizumab such that bleed protection was compromised $(n=2)^{63}$

\section{Conclusion}

Emicizumab is a novel therapeutic antibody that has been shown to be efficacious and well tolerated in several clinical trials, and represents a paradigm shift in the management of HA. ${ }^{9-12}$ While treatment with emicizumab offers HA patients reduced burden of treatment, its incorporation into the clinical landscape requires some adjustments to the well-established monitoring techniques currently used.

Structural and functional differences between emicizumab and FVIII mean that many of the assays most frequently used to monitor the coagulation potential of HA patients are no longer accurate when emicizumab is present in the sample. ${ }^{40}$ Most notably, emicizumab has a considerable shortening effect on the aPTT, rendering many of the most commonly used aPTT-based coagulation assays inaccurate in the presence of even low levels of emicizumab.

To measure the concentration and activity of emicizumab itself, the modified FVIII OSA calibrated against emicizumab offers a cheap and simple method that can be used in the clinical setting to monitor emicizumab plasma concentrations. ${ }^{46}$ With regard to global assessment of hemostatic ability, both ROTEM and the TGA can be used in the presence of emicizumab. ${ }^{48,52}$ Further research is required to confirm the accuracy of ROTEM and TGA in emicizumab-treated patients. Also, their current use is primarily in a research setting; these techniques have not been fully adopted for routine clinical use.

Overall, the measurement of emicizumab in the hemostasis laboratory requires a shift from the functional assays of the past to assays that are specifically tailored for use in patients receiving emicizumab.

\section{Funding}

This study was funded by Roche Pharma AG, Germany.

\section{Conflict of Interest}

J.M. has received honoraria from Bayer, Novo Nordisk, Roche, and Siemens Healthcare Diagnostics for participating in advisory boards and speaker bureaus. I.P. has received honoraria or grants from LFB, Novo Nordisk, Roche, Shire, and Sobi for participating in advisory boards, speaker bureaus, and/or research. B.P. reports no conflicts of interest. B.B. is an employee of Roche and has stock ownership in Roche. J.O. has received honoraria from Bayer, Biogen, Biotest, Chugai, CSL Behring, Grifols, Novo Nordisk, Octapharma, Pfizer, Roche, Shire, and Sobi for participating in advisory boards and speaker bureaus; he has received research funding from Biotest, CSL Behring, Grifols, Novo Nordisk, Octapharma, and Shire. M.S. has received grants from Shire and Pfizer, and personal fees from Roche, Sobi, LFB, Bayer, Shire, Pfizer, Uniqure, Novo Nordisk, and CSL Behring. B.B. reports personal fees from Roche Pharma AG, nonfinancial support from GardinerCaldwell Communications, during the conduct of the study. J.M. reports personal fees from Roche, outside the submitted work.J.O. reports grants and personal fees from Bayer, grants and personal fees from Biotest, personal fees from Chugai, grants and personal fees from CSL Behring, personal fees from Grifols, grants and personal fees from NovoNordisk, grants and personal fees from Octapharma, personal fees from Pfizer, personal fees from Roche, personal fees from SOBI, grants and personal fees from Shire, outside the submitted work. Personal fees were received for travel support, participation in Advisory Boards and participating in symposia as chair or speaker. Dr. M.S. reports nonfinancial support from GardinerCaldwell Communications, during the conduct of the study; grants from Shire, Pfizer, personal fees from Roche, Sobi, LFB, Bayer, Shire, Pfizer, Uniqure, Novo Nordisk, CSL Behring, outside the submitted work. I.P. reports personal fees from Sobi, LFB, Roche, Shire, grants from Novo Nordisk, outside the submitted work.

\section{Acknowledgments}

Medical writing assistance was provided by Sophie Nobes, BSc, of Gardiner-Caldwell Communications.

\section{References}

1 Franchini M, Mannucci PM. Hemophilia A in the third millennium. Blood Rev 2013;27(04):179-184

2 Lai JD, Lillicrap D. Factor VIII inhibitors: advances in basic and translational science. Int J Lab Hematol 2017;39(Suppl 1):6-13

3 Berntorp E. Differential response to bypassing agents complicates treatment in patients with haemophilia and inhibitors. Haemophilia 2009;15(01):3-10

4 Walsh CE, Soucie JM, Miller CH; United States Hemophilia Treatment Center Network. Impact of inhibitors on hemophilia A mortality in the United States. Am J Hematol 2015;90(05): 400-405

5 Food and Drug Administration. HEMLIBRA $®$ (emicizumab-kxwh) injection for subcutaneous use, prescribing information. Initial U.S. approval: 2017. Available at: https://www.accessdata.fda.gov/drugsatfda_docs/label/2017/761083s000lbl.pdf. Accessed January 9, 2019

6 European Medicines Agency. HEMLIBRA® solution for injection: emicizumab pilEa. Initial EU approval: 2018. Available at: https://www.ema.europa.eu/documents/product-information/ hemlibra-epar-product-information_en.pdf. Accessed January 9, 2019 
7 Kitazawa T, Igawa T, Sampei Z, et al. A bispecific antibody to factors IXa and $X$ restores factor VIII hemostatic activity in a hemophilia A model. Nat Med 2012;18(10):1570-1574

8 Sampei Z, Igawa T, Soeda T, et al. Identification and multidimensional optimization of an asymmetric bispecific IgG antibody mimicking the function of factor VIII cofactor activity. PLoS One 2013;8(02):e57479

9 Mahlangu J, Oldenburg J, Paz-Priel I, et al. Emicizumab prophylaxis in patients who have hemophilia A without inhibitors. $\mathrm{N}$ Engl J Med 2018;379(09):811-822

10 Oldenburg J, Mahlangu JN, Kim B, et al. Emicizumab prophylaxis in hemophilia A with inhibitors. N Engl J Med 2017;377(09): 809-818

11 Pipe S, Shima M, Lehle M, et al. A multicentre, open-label, phase 3 study to evaluate the efficacy, safety, and pharmacokinetics of emicizumab prophylaxis administered every 4 weeks in persons with haemophilia A. Lancet Haematol 2019. Doi: https://doi.org/ 10.1016/S2352-3026(19)30054-7

12 Young G, Sidonio RF, Liesner R, et al. Emicizumab prophylaxis provides flexible and effective bleed control in children with hemophilia A with inhibitors: results from the HAVEN 2 Study. 60th Annual Meeting of the American Society of Hematology (ASH) December 1-4, 2018; San Diego, CA

13 Moser KA, Adcock Funk DM. Chromogenic factor VIII activity assay. Am J Hematol 2014;89(07):781-784

14 Teichman J, Chaudhry HR, Sholzberg M. Novel assays in the coagulation laboratory: a clinical and laboratory perspective. Transfus Apheresis Sci 2018;57(04):480-484

15 Kitchen S, Kershaw G, Tiefenbacher S. Recombinant to modified factor VIII and factor IX - chromogenic and one-stage assays issues. Haemophilia 2016;22(Suppl 5):72-77

16 Al-Samkari H, Croteau SE. Shifting landscape of hemophilia therapy: implications for current clinical laboratory coagulation assays. Am J Hematol 2018;93:1082-1090

17 Amiral J, Seghatchian J. Usefulness of chromogenic assays for potency assignment and recovery of plasma-derived FVIII and FIX concentrates or their recombinant long acting therapeutic equivalents with potential application in treated pediatric hemophiliac patients. Transfus Apheresis Sci 2018;57(03): 363-369

18 Kitchen S, Blakemore J, Friedman KD, et al. A computer-based model to assess costs associated with the use of factor VIII and factor IX one-stage and chromogenic activity assays. J Thromb Haemost 2016;14(04):757-764

19 Trossaërt M, Regnault V, Sigaud M, Boisseau P, Fressinaud E, Lecompte T. Mild hemophilia A with factor VIII assay discrepancy: using thrombin generation assay to assess the bleeding phenotype. J Thromb Haemost 2008;6(03):486-493

20 Lyall H, Hill M, Westby J, Grimley C, Dolan G. Tyr346->Cys mutation results in factor VIII:C assay discrepancy and a normal bleeding phenotype - is this mild haemophilia A? Haemophilia 2008;14(01):78-80

21 Pavlova A, Delev D, Pezeshkpoor B, Müller J, Oldenburg J. Haemophilia A mutations in patients with non-severe phenotype associated with a discrepancy between one-stage and chromogenic factor VIII activity assays. Thromb Haemost 2014;111(05): 851-861

22 Peyvandi F, Oldenburg J, Friedman KD. A critical appraisal of onestage and chromogenic assays of factor VIII activity. J Thromb Haemost 2016;14(02):248-261

23 Bowyer A, Key N, Dalton D, Kitchen S, Makris M. The coagulation laboratory monitoring of Afstyla single-chain FVIII concentrate. Haemophilia 2017;23(05):e469-e470

24 Friedman KD, Powell JS, Bensen-Kennedy D. Response: the coagulation laboratory monitoring of AFSTYLA single-chain FVIII concentrate. Haemophilia 2018;24(03):e129-e131

25 Badle S, Barrie A, Spires J, et al. Use of rotational thromboelastometry to monitor boys with severe haemophlia A and inhibitors on treatment with emicizumab. World Congress of the World Federation of Haemophilia (WFH) May 20-24, 2018; Glasgow, Scotland

26 Theusinger OM, Nürnberg J, Asmis LM, Seifert B, Spahn DR. Rotation thromboelastometry (ROTEM) stability and reproducibility over time. Eur J Cardiothorac Surg 2010;37(03):677-683

27 Spiezia L, Bertini D, Boldrin M, et al. Reference values for thromboelastometry (ROTEM ${ }^{\circledR}$ ) in cynomolgus monkeys (Macaca fascicularis). Thromb Res 2010;126(04):e294-e297

28 Durila M. Nonactivated thromboelastometry able to detect fibrinolysis in contrast to activated methods (EXTEM, INTEM) in a bleeding patient. Blood Coagul Fibrinolysis 2016;27(07):828-830

29 Mancuso ME, Fasulo MR. Thrombin generation assay as a laboratory monitoring tool during bypassing therapy in patients with hemophilia and inhibitors. Semin Thromb Hemost 2016;42(01): 30-35

30 Dargaud Y, Luddington R, Gray E, et al. Standardisation of thrombin generation test-which reference plasma for TGT? An international multicentre study. Thromb Res 2010;125(04): 353-356

31 Miller CH. Laboratory testing for factor VIII and IX inhibitors in haemophilia: a review. Haemophilia 2018;24(02):186-197

32 Allain JP, Frommel D. Antibodies to factor VIII. I. Variations in stability of antigen-antibody complexes in hemophilia A. Blood 1973;42(03):437-444

33 Regnault V, Stoltz JF. Quantitation of factor VIII antibodies by an enzyme-linked immunoassay method. Blood 1994;83(04): 1155-1156

34 Sahud MA, Pratt KP, Zhukov O, Qu K, Thompson AR. ELISA system for detection of immune responses to FVIII: a study of 246 samples and correlation with the Bethesda assay. Haemophilia 2007;13(03):317-322

35 Kitazawa T, Esaki K, Tachibana T, et al. Factor VIIla-mimetic cofactor activity of a bispecific antibody to factors IX/IXa and $\mathrm{X} / \mathrm{Xa}$, emicizumab, depends on its ability to bridge the antigens. Thromb Haemost 2017;117(07):1348-1357

36 Uchida N, Sambe T, Yoneyama K, et al. A first-in-human phase 1 study of ACE910, a novel factor VIII-mimetic bispecific antibody, in healthy subjects. Blood 2016;127(13):1633-1641

37 van Dijk K, van der Bom JG, Lenting PJ, et al. Factor VIII half-life and clinical phenotype of severe hemophilia A. Haematologica 2005; 90(04):494-498

38 Knight T, Callaghan MU. The role of emicizumab, a bispecific factor IXa- and factor X-directed antibody, for the prevention of bleeding episodes in patients with hemophilia A. Ther Adv Hematol 2018;9(10):319-334

39 Shima M, Hanabusa H, Taki M, et al. Factor VIII-mimetic function of humanized bispecific antibody in hemophilia A. N Engl J Med 2016;374(21):2044-2053

40 Adamkewicz JI, Chen DC, Paz-Priel I. Effects and interferences of emicizumab, a humanised bispecific antibody mimicking activated factor VIII cofactor function, on coagulation assays. Thromb Haemost 2019;119(07):1084-1093

41 Calatzis A, Chen DC, Paz-Priel I, et al. Effects and interferences of emicizumab - a humanised bispecific antibody mimicking activated factor VIII cofactor function - on coagulation assays. 62nd Annual Meeting of the Gesellschaft für Thrombose- und Hämostaseforschung (GTH) February 20-23, 2018; Vienna, Austria

42 Adamkewicz JI, Soeda T, Kotani N, et al. Effect of emicizumab (ACE910) - a humanized bispecific antibody mimicking FVIIIa cofactor function - on coagulation assays commonly in use for monitoring of hemophilia A patients. Scientific Symposium of the Hemostasis and Thrombosis Research Society (HTRS) April 6-8, 2017; Scottsdale, AZ

43 Muto A, Yoshihashi K, Takeda M, et al. Anti-factor IXa/X bispecific antibody (ACE910): hemostatic potency against ongoing bleeds in a hemophilia A model and the possibility of routine supplementation. J Thromb Haemost 2014;12:206-213 
44 Lenting PJ, Denis CV, Christophe OD. Emicizumab, a bispecific antibody recognizing coagulation factors IX and X: how does it actually compare to factor VIII? Blood 2017;130(23):2463-2468

45 Ferrière $\mathrm{S}$, Lenting PJ. Antibodies in the treatment of haemophilia A-a biochemical perspective. Hamostaseologie 2019;39(01): $36-41$

46 Calhoon W, McInerney M, Calatzis A, et al. Evaluation of a dedicated calibrator and controls for emicizumab quantification. 4th Scientific Meeting of the Thrombosis and Hemostasis Societies of North America (THSNA) April 22-25, 2018; Chicago, IL

47 Jonsson F, Mercier F, Prins NH, et al. Exposure-response modeling of emicizumab for the prophylaxis of bleed counts in hemophilia A patients. 27th Meeting of the Population Approach Group in Europe (PAGE) May 29-June 1, 2018; Montreux, Switzerland

48 Schmitt C, Adamkewicz JI, Xu J, et al. Pharmacokinetics (PK), pharmacodynamics (PD), and PK/PD relationships of emicizumab in persons with hemophilia A (PwHA) with inhibitors from adolescent/adult (HAVEN 1) and paediatric (HAVEN 2) phase 3 studies. 11th Annual Congress of the European Association for Haemophilia and Allied Disorders (EAHAD) February 7-9, 2018; Madrid, Spain

49 Yoneyama K, Schmitt C, Kotani N, et al. A pharmacometric approach to substitute for a conventional dose-finding study in rare diseases: example of phase III dose selection for emicizumab in hemophilia A. Clin Pharmacokinet 2018;57(09):1123-1134

50 Nogami K, Matsumoto T, Tabuchi Y, et al. Modified clot waveform analysis to measure plasma coagulation potential in the presence of the anti-factor IXa/factor X bispecific antibody emicizumab. J Thromb Haemost 2018;16(06):1078-1088

51 Yada K, Nogami K, Kasai R, et al. Enhanced Global Hemostatic Potentials with a Bispecific Antibody to Factors IXa and X (ACE910) in Whole Blood By Rotation Thromboelastometry (ROTEM $\left.{ }^{\circledR}\right)$. 57th Annual Meeting of the American Society of Hematology (ASH) December 5-8, 2015; Orlando, FL

52 Yada K, Nogami K, Kasai R, et al. A novel hemostatic monitoring system convertible to FVIII activity based on non-activated ROTEM (NATEM) for hemophilia A patients during emicizumab prophylaxis. 60th Annual Meeting of the American Society of Hematology (ASH) December 1-4, 2018; San Diego, CA

53 Yada K, Nogami K, Kitazawa T, et al. Mode of enhancement in the global hemostatic potentials with concomitant use of bypassing agents and emicizumab in hemophilia A patients with inhibitor evaluated by ROTEM. 26th Congress of the International Society on Thrombosis and Haemostasis (ISTH) July 11, 2017; Berlin, Germany

54 Soeda T, Kitazawa T, Muto A, et al. In vitro characterization of ACE910, a humanized bispecific antibody to factors IXa and X. World Congress of the World Federation of Haemophilia (WFH) May 11-15, 2014; Melbourne, Australia

55 Adamkewicz JI, Muto A, Soeda T, et al. In vitro thrombin generation testing and in vivo venous stasis model for assessing the pro-coagulant effects of FVIII, emicizumab, rFVIIa, and aPCC. 10th Annual Congress of the European Association for Haemophilia and Allied Disorders (EAHAD) February 1-3, 2017; Paris, France

56 Dargaud Y, Lienhart A, Janbain M, Le Quellec S, Enjolras N, Negrier C. Use of thrombin generation assay to personalize treatment of breakthrough bleeds in a patient with hemophilia and inhibitors receiving prophylaxis with emicizumab. Haematologica 2018; 103(04):e181-e183

57 Calatzis A, Kotani N, Levy GG, et al. Effect of emicizumab (ACE910) - a humanized bispecific antibody mimicking FVIII cofactor function - on a variety of assay systems. 1st European Congress on Thrombosis and Haemostasis (ECTH) September 28-30, 2016; The Hague, The Netherlands

58 Nogami K, Soeda T, Matsumoto T, Kawabe Y, Kitazawa T, Shima M. Routine measurements of factor VIII activity and inhibitor titer in the presence of emicizumab utilizing anti-idiotype monoclonal antibodies. J Thromb Haemost 2018;16(07):1383-1390

59 Adamkewicz JI, Kim B, Steinbuesch D, et al. Measurement of FVIII Inhibitor Titer Using a Chromogenic Bethesda Assay (CBA) in the Presence of Emicizumab (ACE910), a Humanized Bispecific Antibody Mimicking FVIIIa Cofactor Function. Scientific Symposium of the Hemostasis and Thrombosis Research Society (HTRS) April 6-8, 2017; Scottsdale, AZ

60 Adamkewicz JI, Schmitt C, Asikanius E, et al. Pharmacodynamic data and coagulation biomarkers in person with hemophilia $A$ with inhibitors: results from the HAVEN 1 emicizumab phase 3 study. 26th Congress of the International Society on Thrombosis and Haemostasis (ISTH) July 8-13, 2017; Berlin, Germany

61 Sadeghi-Kohmami A, Boylan M, Chen DC, et al. Agreement between a chromogenic modified Nijmegen-Bethesda assay and a qualitative ELISA test in detection of factor VIII inhibitors in plasma from persons with hemophilia A (PwHA). World Congress of the World Federation of Hemophilia (WFH) May 20-24, 2018; Glasgow, Scotland

62 (CHMP) CfMPfHU. Guideline on Immunogenicity Assessment of Therapeutic Proteins. Amsterdam, the Netherlands: European Medicines Agency; 2017

63 Paz-Priel I, Chang T, Asikanius E, et al. Immunogenicity of emicizumab in people with hemophilia A (PwHA): results from the HAVEN 1-4 studies. 60th Annual Meeting of the American Society of Hematology (ASH) December 1-4, 2018; San Diego, CA

64 Ahmad SS, London FS, Walsh PN. Binding studies of the enzyme (factor IXa) with the cofactor (factor VIIla) in the assembly of factor-X activating complex on the activated platelet surface. J Thromb Haemost 2003;1(11):2348-2355

65 Lapan KA, Fay PJ. Localization of a factor X interactive site in the A1 subunit of factor VIIIa. J Biol Chem 1997;272(04):2082-2088

66 Lenting PJ, Donath MJ, van Mourik JA, Mertens K. Identification of a binding site for blood coagulation factor IXa on the light chain of human factor VIII. J Biol Chem 1994;269(10):7150-7155 